M. Koike

Nagoya Math. J.

Vol. 98 (1985), 109-115

\title{
HIGHER RECIPROCITY LAW, MODULAR FORMS OF WEIGHT 1 AND ELLIPTIC CURVES
}

\author{
MASAO KOIKE
}

\section{§. Introduction}

In this paper, we study higher reciprocity law of irreducible polynomials $f(x)$ over $\boldsymbol{Q}$ of degree 3, especially, its close connection with elliptic curves rational over $\boldsymbol{Q}$ and cusp forms of weight 1 . These topics were already studied separately in a special example by Chowla-Cowles [1] and Hiramatsu [2]. Here we bring these objects into unity.

Let

$\mathscr{C}_{0}=$ the set of number fields $K$ over $\boldsymbol{Q}$ such that

(1) $K$ is a Galois extension over $\boldsymbol{Q}$ with $\mathrm{Gal}(K / \boldsymbol{Q}) \cong S_{3}$, the symmetric group of degree 3 ,

(2) $K$ contains an imaginary quadratic field $k$.

For any $K$ in $\mathscr{C}_{0}$, we can associate three other objects: (1) $f(x)$ : irreducible polynomials over $\boldsymbol{Q}$ of degree 3, (2) $F(\tau)$ : cusp forms of weight 1, (3) $E$ : elliptic curves rational over $\boldsymbol{Q}$; let

$$
\begin{aligned}
\mathscr{C}_{1}= & \text { the set of all irreducible polynomials } f(x) \text { over } \boldsymbol{Q} \text { of degree } 3 \\
& \text { whose splitting field } K_{f} \text { over } \boldsymbol{Q} \text { belongs to } \mathscr{C}_{0} . \\
\mathscr{C}_{2}= & \text { the set of all normalized cusp forms } F(\tau) \text { of weight } 1 \text { on } \Gamma_{0}(N) \\
& \text { whose Mellin transform is } L \text {-function with an ideal character } \\
& \chi \text { of degree } 3 \text { of imaginary quadratic field } k \text { and the abelian } \\
& \text { extension } K_{F^{\prime}} \text { over } k \text { which corresponds to the kernel of } \chi \\
& \text { belongs to } \mathscr{C}_{0} . \\
\mathscr{C}_{3}= & \text { the set of all elliptic curves } E \text { rational over } \boldsymbol{Q} \text { such that the } \\
& \text { field } E_{2} \text { generated by coordinates of } 2 \text {-division points on } E \\
& \text { belongs to } \mathscr{C}_{0} .
\end{aligned}
$$


Therefore we can define maps $\varphi_{i}: \mathscr{C}_{i} \rightarrow \mathscr{C}_{0}(i=1,2,3)$ as follows;

$$
\varphi_{1}(f)=K_{f}, \quad \varphi_{2}(F)=K_{F}, \quad \varphi_{3}(E)=E_{2} .
$$

For any $K$ in $\mathscr{C}_{0}$, let $f(x) \in \varphi_{1}^{-1}(K), F(\tau) \in \varphi_{2}^{-1}(K)$ and $E \in \varphi_{3}^{-1}(K)$. Then our theorems give

(I) the relation between the higher reciprocity law of $f(x)$ and Fourier coefficients of $F(\tau)$, which is called the arithmetic congruence relation.

(II) the relation between the higher reciprocity law of $f(x)$ and $L$-function of $E$.

(III) congruences modulo 2 between $F(\tau)$ and $L$-function of $E$.

These results are a generalization of an example given in [1] and [2].

The author would like to express his hearty thanks to Prof. Hiramatsu for giving him a lecture on this subject and invaluable conversation.

\section{§1. Proof of (I)}

Hereafter we fix $K$ in $\mathscr{C}_{0}$. Let $f(x)=a x^{3}+b x^{2}+c x+d$ be an element in $\varphi_{1}^{-1}(K)$. Let $M$ be the product of all primes which appear in $a, b, c$ and $d$.

For any prime $p, p \nmid M$, put $f_{p}(x)=f(x) \bmod p$. Then $f_{p}(x)$ is a polynomial over $\boldsymbol{F}_{p}$, the finite field with $p$ elements, of degree 3 . We define Spl $\{f(x)\}$ to be the set of primes such that the polynomial $f_{p}(x)$ factors into a product of distinct linear polynomials over $\boldsymbol{F}_{p}$. By the higher reciprocity law for $f(x)$, we mean a rule to determine the set Spl $\{f(x)\}$ up to finite set of primes.

Let $F(\tau)=\sum_{n=1}^{\infty} a(n) e[n \tau], e[\tau]=\exp (2 \pi \sqrt{-1} \tau)$, be a normalized cusp form of weight 1 in $\varphi_{2}^{-1}(K)$. Let $\chi$ be the non-trivial ideal character of $k$ corresponding to the abelian extension $K$ over $k$. Let $-D$ and $f$ denote the discriminant of $k$ and the conductor of $\chi$. Then

$$
L(s, \chi)=\sum_{n=1}^{\infty} a(n) n^{-s}
$$

and $F(\tau)$ is a cusp form of weight 1 on $\Gamma_{0}(D N f)$ with the character $(-D / *)$ where $N \mathfrak{f}$ denotes the norm of $\mathfrak{f}$ on $k$ over $\boldsymbol{Q}$. Let $\rho$ denote the complex conjugation. From the assumption, it follows that $\chi(\mathfrak{a})^{\rho}=\chi\left(\mathfrak{a}^{\rho}\right)$ for any integral ideal $a$ of $k$. 
THEOREM 1 (arithmetic congruence relation). Let $p$ be any prime such

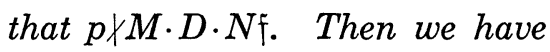

$$
\#\left\{\alpha \in \boldsymbol{F}_{p} \mid f_{p}(\alpha)=0\right\}=a(p)^{2}-\left(\frac{-D}{p}\right) .
$$

Proof. The proof is similar to that of Theorem 2 in [2]. Let $p$ be a prime as above. It is easily seen that

$$
\begin{aligned}
a(p)=0 \Longleftrightarrow & (-D / p)=-1, \\
\Longleftrightarrow & \text { the splitting field of } f_{p}(x) \text { over } \boldsymbol{F}_{p} \text { is a quadratic ex- } \\
& \text { tension over } \boldsymbol{F}_{p}, \\
\Longleftrightarrow & f_{p}(x) \text { has exactly } 1 \text { linear factor over } \boldsymbol{F}_{p} .
\end{aligned}
$$

Now we assume that $(-D / p)=1$. Then $p$ decomposes into a product of two prime ideals $\mathfrak{p}$ and $\mathfrak{p}^{\prime}$ where $\mathfrak{p}^{\prime}$ is the conjugate of $\mathfrak{p}$. It is clear that

$$
\begin{aligned}
a(p)=2 & \Longleftrightarrow \chi(\mathfrak{p})=1, \\
& \Longleftrightarrow p \text { splits completely in } K, \\
& \Longleftrightarrow f_{p}(x) \text { has exactly } 3 \text { distinct linear factors over } F_{p} .
\end{aligned}
$$

And also it is clear that

$$
\begin{aligned}
a(p)=-1 & \Longleftrightarrow \chi(p)=\omega, \text { a non-trivial cube root of unity, } \\
\Longleftrightarrow & \text { remains prime in } K . \\
& \text { the splitting field of } f_{p}(x) \text { over } F_{p} \text { is a cubic exten- } \\
& \text { sion over } \boldsymbol{F}_{p}, \\
\Longleftrightarrow & f_{p}(x) \text { has no linear factor over } \boldsymbol{F}_{p} .
\end{aligned}
$$

Summarizing these results, we obtain a proof of Theorem 1. Q.E.D.

Corollary 1. Sp1 $\{f(x)\}$ coincides with the set

$$
\{p: \text { prime } \mid p \nmid M \cdot D \cdot N \mathfrak{F}, a(p)=2\}
$$

up to finite set of primes.

Proof. This is obvious from Theorem 1.

Q.E.D.

\section{§2. Proof of (II)}

Let $E$ be an elliptic curve rational over $\boldsymbol{Q}$ in $\varphi_{3}^{-1}(K)$, which is defined by $y^{2}=f(x)$ where $f(x)$ is a polynomial of degree 3 over $Q ; f(x)=a x^{3}+$ $b x^{2}+c x+d, a, b, c, d \in \boldsymbol{Q}$. Let $N$ denote the conductor of $E$ over $\boldsymbol{Q}$. Let $E_{2}$ denote the field generated by the coordinates of 2-division points on $E$ 
over $\boldsymbol{Q}$. Then $E_{2}$ coincides with the splitting field of $f(x)$ over $\boldsymbol{Q}$. Let $\boldsymbol{p}$ be an odd prime such that $p \nmid N$, and let $\tilde{E}_{p}$ denote the reduction modulo $p$ of $E$ which is an elliptic curve over $F_{p}$. Let $N_{p}=N_{p}(E)$ denote the number of $\boldsymbol{F}_{p}$-rational points of $\tilde{E}_{p}$. Further we assume that $p$ is prime to $M D N\left\lceil\right.$ as in Section 1 , and put $f_{p}(x)=f(x) \bmod p$. Then we can prove

LEMMA 1. With the notation as above, we have (*)

$$
N_{p}-1 \equiv \#\left\{\alpha \in F_{p} \mid f_{p}(\alpha)=0\right\} \quad(\bmod 2) .
$$

Proof. The proof was given in a special case in [1], but for the completeness of the paper, we give here the proof in detail. It is known that the number of solutions of $y^{2} \equiv f(x)(\bmod p)$ in $F_{p}^{2}$ is equal to $N_{p}-1$. We notice that the right hand side of $(*)$ is odd if and only if $f_{p}(x)$ has at least one linear factor over $F_{p}$. And, it is clear that $f_{p}(x)$ has a linear factor if and only if the number of solutions of $y^{2} \equiv f(x)(\bmod p)$ is odd.

Q.E.D.

Theorem 2. With the notation as above, we have the following equivalences:

(1) $f_{p}(x)$ has exactly one linear factor over $\boldsymbol{F}_{p}$ if and only if $N_{p}-1$ is odd and $(-D / p)=-1$.

(2) $f_{p}(x)$ is irreducible over $F_{p}$ if and only if $N_{p}-1$ is even and $(-D / p)=1$.

(3) $f_{p}(x)$ has three distinct linear factors over $\boldsymbol{F}_{p}$ if and only if $N_{p}-1$ is odd and $(-D / p)=1$.

Proof. (2) is obvious from Lemma 1. (1) is already proved in the proof of Theorem 1. Hence (3) is also proved.

Q.E.D.

Remark 1. The Galois group of $E_{2}$ over $\boldsymbol{Q}$ is isomorphic to $S_{3}$ if and only if $E$ has no $Q$-rational points of order 2 and the discriminant of $E$ is not square.

Remark 2. We should remark that, in the proofs of Lemma 1 and Theorem 2, we need not use the condition that $K_{f}\left(=E_{2}\right)$ contains an imaginary quadratic field. This condition is needed only for assuring the existence of cusp forms of weight 1.

Remark 3. Let $E, E^{\prime}$ be in $\varphi_{3}^{-1}(K)$. Let $N$ and $N^{\prime}$ denote the conductors of $E$ and $E^{\prime}$. Let $p$ be any odd prime such that $p \nmid N N^{\prime}$. Then Lemma 1 shows that, for almost all $p$, 


$$
N_{p}(E) \equiv N_{p}\left(E^{\prime}\right) \quad(\bmod 2)
$$

\section{§3. Proof of (III)}

Let $E$ be in $\varphi_{3}^{-1}(K)$ and $F(\tau)=\sum_{n=1}^{\infty} a(n) e[n \tau]$ in $\varphi_{2}^{-1}(K)$. We use same notation as in Section 1 and Section 2. Combining Theorem 1 and Theorem 2, we obtain

THEOREM 3. Let $p$ be any odd prime such that $p \nmid N M D N F .$, Then we have

$$
N_{p}(E) \equiv a(p) \quad(\bmod 2) .
$$

For elliptic curves rational over $\boldsymbol{Q}$, there is a famous Taniyama-Weil conjecture. If we assume this conjecture, for the elliptic curve $E$ in Section 2, there exists the normalized cusp form $G(\tau)=\sum_{n=1}^{\infty} c(n) e[n \tau]$ of weight 2 on $\Gamma_{0}(N)$ such that

$$
N_{p}(E)=1+p-c(p), \quad \text { for any prime } p, p \nmid N .
$$

Hence, we get

CoROLlaRY. With the above assumption, we get the congruence mod 2 between $F(\tau)$ and $G(\tau)$ :

$$
c(p) \equiv a(p) \quad(\bmod 2)
$$

for any odd prime $p$, such that $p \nmid N M D N \mathfrak{F}$.

Remark. In a special example treated in [1], this type of congruences $\bmod 2$ means that

$$
\eta(\tau)^{2} \eta(11 \tau)^{2} \equiv \eta(2 \tau) \eta(22 \tau) \quad(\bmod 2),
$$

which follows easily from the fact, $(1-x)^{2} \equiv 1-x^{2}(\bmod 2)$.

$\S 4$.

Let $F(\tau)=\sum_{n=1}^{\infty} a(n) e[n \tau]$ be an element in $\mathscr{C}_{2}$. We assume that there exists a cusp form $H(\tau)=\sum_{n=1}^{\infty} b(n) e[n \tau]$ of weight 2 satisfying

(1) $H(\tau)$ is a normalized primitive cusp form,

(2) $b(n) \in Z$ for all $n \geqq 1$,

(3) For almost all primes $p, a(p) \equiv b(p)(\bmod 2)$.

By the assumptions (1) and (2), there exists an elliptic curve $E$ defined over $\boldsymbol{Q}$ associated with $H(\tau)$ as in Section 3 . 
THEOREM 4. Under the above assumption, we have

$$
K_{F}=E_{2} \text {. }
$$

Namely, $E$ belongs to $\mathscr{C}_{3}$ and $\varphi_{3}(E)=\varphi_{1}(F)$.

Proof. We denote the defining equation of $E$ by $y^{2}=g(x)$ where $g(x)$ is a polynomial over $\boldsymbol{Q}$ of degree 3. For any good prime $p$ for $E$, let $N_{p}$ denote the number of $\boldsymbol{F}_{p}$-rational points of the reduction $\bmod p$ of $E$. Then the assumption (3) shows that

$$
N_{p} \equiv a(p)(\bmod 2), \quad \text { for almost all odd, good primes . }
$$

Put $T_{1}=\{p:$ good prime $\mid a(p)=2\}, \quad T_{2}=\{p: \operatorname{good}$ prime $\mid a(p)=0\}$, and $T_{3}=\{p:$ good prime $\mid a(p)=-1\}$. Applying Tchebotarev density theorem to $K_{F}$, we know that the densities of $T_{1}, T_{2}$ and $T_{3}$ are $1 / 6,1 / 2$ and $1 / 3$ respectively. The above congruence shows that $T_{3}=\left\{p:\right.$ prime $\mid N_{p}$ is odd $\}$ up to finite set of primes.

If $g(x)$ is reducible over $\boldsymbol{Q}, N_{p}$ is even for any good prime; this contradicts the above result. Hence $g(x)$ is irreducible over $\boldsymbol{Q}$. We assume that the splitting field $K_{g}$ of $g(x)$ is abelian over $\boldsymbol{Q}$. Then the densities of sets of primes $U_{1}=\left\{p\right.$ : prime $\mid g_{p}(x)$ is a product of linear factors over $\left.\boldsymbol{F}_{p}\right\}$ and $U_{2}=\left\{p\right.$ : prime $\mid g_{p}(x)$ is irreducible over $\left.\boldsymbol{F}_{p}\right\}$ are $1 / 3$ and $2 / 3$ respectively; this contradicts the above result. Hence $\left[K_{g}: Q\right]=6$. Let $k^{\prime}$ denote the quadratic field contained in $K_{g}$. We assume that $k \neq k^{\prime}$, Let $(k / p)$ denote the Kronecker symbol. Then $(k / p)=-1$ induces $a(p)=0$, hence $N_{p}$ is even. Also $\left(k^{\prime} / p\right)=-1$ induces that $N_{p}$ is even. Since $k \neq k^{\prime}$, the density of the set of primes $\left\{p\right.$ : prime $\mid(k / p)=-1$ or $\left.\left(k^{\prime} / p\right)=-1\right\}$ is $3 / 4$; this contradicts the above result. Hence $K_{g} \supset k$. Since $K_{f} / k$ and $K_{g} / k$ are abelian extensions and the decomposition rule of primes of $k$ in $K_{f}$ and $K_{g}$ coincides to each other, we get $K_{f}=K_{g}$.

Q.E.D.

\section{REFERENCES}

[1] S. Chowla and M. Cowles, On the coefficients $c_{n}$ in the expansion $x \prod_{n=1}^{\infty}\left(1-x^{n}\right)^{2}$ $\left(1-x^{11 n}\right)^{2}=\sum_{1}^{\infty} c_{n} x^{n}$, J. reine angew. Math., 292 (1977), 115-116.

[2] T. Hiramatsu, Higher reciprocity law and modular forms of weight one, Comm. Math. Univ. St. Paul, 31 (1982), 75-85.

[ 3 ] T. Hiramatsu and Y. Mimura, The modular equation and modular forms of weight one, preprint.

[4] T. Hiramatsu, N. Ishii and Y. Mimura, On indefinite modular forms of weight one, preprint. 
[ 5 ] C. Moreno, The higher reciprocity law: an example, J. Number Theory, 12 (1980), $57-70$.

Department of Mathematics

Nagoya University

Chikusa-ku, Nagoya 464

Japan 